\title{
THOMAS PARIS
}

CNRS (GREGHEC) ; HEC Paris

\section{DAVID MASSÉ}

i3-SES, Télécom Paris ; Institut polytechnique

de Paris

\section{Le management des industries créatives}

\section{Un paradigme spécifique et des configurations organisationnelles variées}

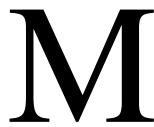

anagement et création... Le bel oxymore ! L'association de ces termes heurte nos conceptions, car elle renvoie spontanément aux idées d'un côté de standardisation, de stabilité, de contrôle, et de l'autre de singularité, de transgression ou d'incertitude. Cette opposition sémantique ne peut cependant pas masquer que ces univers cohabitent et se sont rapprochés, notamment depuis l'introduction du terme de creative industries $^{1}$ par Richard Caves (2000) et des recherches nombreuses, mais fragmentées - qui se sont attachées à mieux comprendre l'organisation et le management de ces industries dans une grande variété d'approches théoriques et méthodologiques. Cet article introductif entend affirmer ou réaffirmer la pertinence de la

1. Que nous avons pris l'habitude de traduire, abusivement, par « industries créatives ». Dans le monde anglo-saxon, le terme d'industrie renvoie à la notion de secteur économique, et en inclut, comme le théâtre ou les arts plastiques, qui n'ont pas de dimension industrielle. La qualification de créative peut quant à elle laisser supposer ces secteurs auraient le monopole de la créativité. On trouve de la créativité dans beaucoup d'autres domaines, et toutes les organisations de ces secteurs ne sont pas créatives. Une traduction plus juste serait « secteurs de création ». 
catégorie d' « industries créatives » vis-à-vis des recherches en management. Il développe l'idée que les entreprises qui évoluent dans ces industries font face à un paradigme managérial idiosyncratique qui repose sur trois spécificités structurantes : différenciation par l'originalité, abondance, subjectivité du créateur.

La catégorie est pertinente, et des secteurs qui peuvent paraître aux antipodes les uns des autres - jeu vidéo et parfum, grande cuisine et musique, édition et mode, spectacle vivant et architecture... - ont un ADN commun. Que la catégorie soit pertinente ne doit pas occulter pour autant sa variété. Elle apparaît, dans l'énumération qui vient d'être faite, dans les modes de diffusion et dans les matériaux de création, qui structurent cette agrégation d'industries. Elle se manifeste autour de deux autres lignes de faille. D'une part, si ces entreprises s'inscrivent dans un paradigme managérial spécifique, elles le font de manière plus ou moins volontariste, plus ou moins consciente, donnant lieu au sein de ces industries à des «mondes opposés » qui peuvent avoir tendance à se polariser. D'autre part, même si l'on retrouve un paradigme managérial commun à ces entreprises, il se manifeste dans des contextes différents, que nous synthétisons à travers trois grandes configurations organisationnelles (direction, orchestration, facilitation).

\section{I - GENÈSE ET DÉVELOPPEMENT DU CONCEPT}

$\mathrm{Au}$ cours des dernières décennies, les industries de la création ont fait l'objet d'une attention importante, tant dans le monde académique que dans ceux des entreprises et des politiques publiques.

\section{Une catégorie qui porte des ambitions politiques}

Le monde politique s'est intéressé aux industries de la création à travers la publication de nombreux rapports (DCMS, 2001, 1998 ; KEA, 2009 ; UNCTAD, 2010) qui ont défendu l'idée que ces secteurs pouvaient être moteurs de la croissance dans les économies occidentales, s'inscrivant ainsi dans un engouement autour de l'économie de la connaissance ou l'économie de l'immatériel (Camdessus, 2004 ; Cohen et Verdier, 2008 ; Lévy et Jouyet, 2006). L'Australie, pionnière, a proposé une catégorie ad hoc agrégeant un ensemble de secteurs liés à la création dans un rapport du ministère des Communications et des Arts (Department of Communications and the Arts, 1994). L'objectif de ce rapport commandé par le gouvernement était de faire valoir le potentiel économique de l'activité culturelle. Cette idée a été reprise au RoyaumeUni, avec un impact plus large, dans un rapport du ministère de la Culture, des Médias et du Sport, lequel proposait une définition de ces industries dites « créatives »: «ces industries qui trouvent leur origine dans la créativité, les compétences et le talent d'une personne et qui ont un fort potentiel de croissance et d'emploi à travers la production et l'exploitation de la propriété intellectuelle » ${ }^{2}$ (DCMS, 1998, p. 4). Cette définition englobait un large éventail de secteurs, incluant le design, la publicité,

2. "those industries which have their origin in individual creativity, skill and talent and which have a potential for wealth and job creation through the generation and exploitation of intellectual property" (DCMS, 1998, p. 4). 
l'architecture, les arts de la scène, les jeux vidéo, l'artisanat, la mode, la musique, le cinéma, la télévision, la radio, l'édition et le logiciel interactif. Dans ce contexte, l'engouement des politiques autour de ces industries s'explique par leur croissance réelle ou escomptée (UNCTAD, 2010), leur poids relatif dans l'économie (France Créative, 2013 ; UNCTAD, 2010), mais aussi par leur impact sur l'innovation dans les industries plus traditionnelles (KEA, 2009 ; UNCTAD, 2010).

\section{Des régularités économiques et une multiplication des travaux académiques}

Dans le monde académique, la notion a été introduite par Richard Caves dans un livre intitulé Creative Industries: Contracts Between Art and Commerce. Par cette appellation, Caves (2000) contribue à la caractérisation de ces industries et met en évidence des régularités dans leur économie, les problématiques auxquelles elles sont confrontées et les réponses qui leur sont apportées. Ces travaux prolongent les réflexions initiées dans les années 1970 par Howard Becker sur la dimension organisée des «mondes de l'arts » et ouvrent de nouvelles pistes de recherches pour le management. À partir des années 2000, de nombreux travaux ont exploré ces industries, dans leur variété : la publicité (Moeran, 2009), l'architecture (Jones et Livne Tarandach, 2008), la parfumerie (Endrissat et al., 2016), le design (Verganti, 2003), le cinéma (Cattani et al., 2008), la musique (Benghozi et Paris, 2003 ; Thompson et al., 2007), les arts de la scène (Glynn, 2000 ; Agid et Tarondeau, 2003 ; Massé et Paris, 2013), la grande cuisine (Bouty et Gomez, 2010 ;
Durand et al., 2007 ; Svejenova et al., 2010), les jeux vidéo (Cohendet et Simon, 2007 ; Tschang, 2007 ; Lê et al., 2013 ; Chiambaretto et al., 2019), etc. Ces travaux ont nourri des thématiques diverses (développement de produits/innovation, open innovation, écosystèmes, etc.) tout en contribuant à de multiples champs théoriques : communautés, théorie des ressources, néo-institutionnalisme, développement de nouveaux produits, business models, habitus, créativité... L'engouement nouveau autour de ces secteurs s'est traduit par l'apparition de sous-thématiques dédiées dans les conférences de management et par la multiplication des numéros spéciaux de revues.

\section{Exotiques ou exemplaires ? Des sources d'inspiration pour le management}

Le monde des entreprises a longtemps considéré ces industries comme "exotiques », c'est-à-dire comme évoluant à l'extérieur de la sphère économique ou mettant en avant des spécificités trop importantes pour que puissent en être tirés des enseignements utiles. La place considérée comme décisive accordée à des individus singuliers, «talents» ou « génies », a été pour beaucoup dans cette mise à l'écart, faisant écho à ce que Becker (1974) avait qualifié, pour mieux en nier la réalité, de mythe de l'auteur romantique. Les temps ont changé et ces industries, dans un remarquable mouvement de balancier, sont aujourd'hui perçues comme une source d'inspiration pour de nombreuses entreprises, témoignant par là sans doute d'une substitution d'une vision mécaniste de la création, basée sur 
des recettes éprouvées, à la vision romantique basée sur le génie individuel. Cet intérêt tient à ce que leur savoir-faire apporte des réponses à des problématiques très actuelles pour beaucoup d'entreprises : la conduite de projet, l'innovation répétée, l'innovation de rupture, la créativité, le management des talents, la gestion du risque... La multiplication d'évènements, de moments et d'espaces d'échanges et de rencontres entre le monde de la création et celui des affaires (Séminaire Création de l'École de Paris du management, C2 Montréal, École d'été en management de la création et de l'innovation de Mosaic-HEC Montréal, etc.) témoigne de cet engouement.

Cependant, malgré cet intérêt croissant et après deux décennies de recherches sur ces secteurs, force est de constater que, à part quelques tentatives (Bilton, 2006 ; Paris, 2010, 2013), les travaux sur les industries créatives ne vont pas au-delà de contributions fragmentées à ces multiples champs théoriques, qu'elles restent encore souvent cantonnées à l'une de ces industries, et qu'elles ont du mal à se distinguer ou s'affranchir des travaux sur la créativité (Amabile, 1988) ou la créativité organisationnelle (Woodman et al., 1993). Tout juste se limitent-ils à souligner, comme élément fédérateur, que le management dans ces secteurs est confronté à des tensions et à des paradoxes (Lampel et al., 2000). La notion de creatives industries n'est toujours pas assumée en tant qu'objet homogène, et il n'y a pas de théorie des industries créatives, alors même qu'elles s'inscrivent dans un paradigme spécifique que nous détaillons plus loin, sur les plans économique et managérial.
Le développement des travaux sur ces secteurs s'est parfois accompagné de la revendication du caractère avant-gardiste des pratiques de management des industries créatives, et de leur diffusion dans d'autres industries (Benghozi, 1995; Cohendet et al., 2010). Cet argument est corroboré par le constat du caractère génétique ou intrinsèque, dans ces industries, de pratiques qui se sont répandues dans les autres secteurs : de même que M. Jourdain faisait de la prose sans le savoir, les industries créatives pratiquaient la gestion de projet ou l'innovation ouverte bien avant qu'elles ne s'imposent comme incontournables dans les autres secteurs.

De là à considérer que ces industries constituent le laboratoire de nouvelles pratiques de management, il n'y a qu'un pas, qui, chacun voit midi à sa porte, a été parfois franchi. Or il nous faut bien admettre, d'une part, que toutes les entreprises de ces secteurs ne présentent pas le même degré d'innovation ou de créativité ; d'autre part, que le phénomène contraire s'observe aussi, les différentes modes managériales (Midler, 1986) qui se déploient dans les industries traditionnelles influençant aussi les démarches et outils de management des industries créatives.

\section{II - UN PARADIGME SPÉCIFIQUE À L'ACTIVITÉ DE CRÉATION}

Caves (2000) a avancé l'idée d'une économie spécifique aux industries de la création, qu'il a déclinée autour de sept propriétés : l'incertitude de la demande (nobody knows property), l'art pour l'art (art for art's sake), qui renvoie à l'existence de motivations spécifiques chez les travailleurs de ces secteurs, la mobilisation d'équipages 
hétéroclites pour produire les biens (motley crew), la variété infinie (infinite variety) de biens dont on recherche la différenciation, la hiérarchie des talents (A list/B list) selon laquelle le marché des talents se structure en deux niveaux, la fuite du temps (time flies) dans le déroulement des projets et la durabilité des produits (ars longa) qui peut engendrer des rentes durables pour leurs créateurs. Ces principes, en réalité, renvoient à un régime spécifique qui a des répercussions tant sur l'économie de ces secteurs - on peut parler d'une économie de la création - que des modalités managériales qui y ont cours.

\section{Différenciation par l'originalité et abondance}

L'activité de création consiste à concevoir des produits nouveaux sans critère de valeur objectif. On peut mesurer, durant sa conception, les performances d'un nouveau véhicule, que ce soit en matière de vitesse, d'émissions de $\mathrm{CO}_{2}$, de bruit ou de sécurité. On ne peut pas définir des critères objectifs de qualité pour un roman, une chanson, un jeu vidéo ou un parfum, avant leur mise sur le marché. Dès lors, l'originalité s'affirme comme une valeur cardinale, recherchée dans un équilibre avec une notion de qualité qui s'exprime, dans la phase de réalisation de la proposition, par l'idée de « fonctionner » ou « ne pas fonctionner ». On dira par exemple qu'un roman fonctionne, sans que cela ne préjuge en rien de son succès futur sur le marché.

Différenciation et subjectivité constituent ainsi les piliers sur lesquels se bâtit l'économie de la création. L'abondance et l'hyperconcurrence en sont des conséquences : dans le jeu de la différenciation, les acteurs établis n'ont pas d'avantage compétitif $^{3}$, au contraire même, puisqu'ils auront tendance à se donner des contraintes qui tiennent à leur histoire et à leurs clients, quand de nouveaux acteurs seront plus à même de casser les codes et faire des propositions iconoclastes. En outre, l'originalité se révèle beaucoup mieux dans l'abondance. Quant à la valeur de la proposition, elle sera établie par la rencontre avec le marché : en l'absence de critère objectif, c'est cette rencontre qui constituera le juge de paix. Becker (1982) exprimait cela en indiquant que la valeur dans les mondes de l'art était un construit social.

\section{Subjectivité du créateur}

Si l'on projette ces caractéristiques dans les entreprises, cela donne lieu à un paradigme singulier (Paris, 2010). Créer consiste à concevoir des produits en l'absence de repères pour un mode de diffusion spécifique. Cette absence de repères s'avère structurante. Elle se traduit par le poids du risque et l'enjeu pour les individus en charge des projets d'évoluer dans cet environnement incertain (Menger, 2009). Elle se traduit aussi par l'acceptation de l'échec, pendant de l'abondance et de l'hyperconcurrence, ainsi que par l'importance accordée à la subjectivité individuelle : dans la mesure où il n'y a pas de critère objectif pour choisir entre deux propositions, l'essentiel est de garantir la cohérence d'ensemble du projet, dont un porteur unique est le garant. «Quand je n'ai pas de bleu, je mets du rouge » disait

3. Leur avantage compétitif repose sur leur réputation, forgée par leurs réalisations passées. 
Picasso. Quand un mode de décision fondé sur le consensus ou les études de marché est pertinent dans un contexte où l'on accepte les repères, il l'est beaucoup moins lorsqu'il s'agit de se mettre en position d'offrir une proposition nouvelle. En cela, la frontière pertinente n'est pas celle qui sépare l'innovation de la création, mais celle qui sépare les activités de conception avec repères des activités qui s'affranchissent de tous repères ${ }^{4}$. Nous pouvons parler de « conception subjectivée » pour désigner ce régime qui met en avant une absence de repères relatifs au marché, au profit de la subjectivité du créateur pour guider les choix de conception.

\section{Un paradigme plus ou moins assumé, source de polarisation}

Si cette absence de repères est un élément distinctif, la réalité est plus nuancée, beaucoup d'entreprises s'écartant, plus ou moins consciemment, de ce refus des repères. Une tendance naturelle pour beaucoup est même de minimiser le risque, par différents moyens qui visent à se rapprocher du marché (recours à des tests, des études de marché, des recettes, des règles, etc.). Entre celles qui assument parfaitement cette absence de repères et celles qui ne le font pas, les entreprises peuvent donc appartenir à des «mondes opposés » (Paris et al., 2020), proposant des produits différents et reposant sur des acteurs différents : les films indépendants contre les films commerciaux, la haute cuisine contre l'industrie alimentaire ou la haute couture contre l'industrie de la mode sont des exemples de polarisations que l'on retrouve dans nombre de secteurs constitutifs des industries créatives.

\section{III - TROIS CONFIGURATIONS ORGANISATIONNELLES}

Cette dernière remarque nous conduit aussi à considérer que les industries créatives, audelà de leur socle commun, mettent aussi en avant des différences dans la manière de gérer l'activité de création, propres à des contextes spécifiques (Mainemelis et al., 2015, Paris, 2013). Un élément de différenciation porte sur les configurations organisationnelles qui se distinguent selon la place du ou des créateurs dans ou à l'extérieur de l'organisation (AbecassisMoedas et Benghozi, 2012) et des types de ressources intégrées ou non par l'entreprise (Paris, 2013). À titre d'exemple, dans le domaine de l'animation, un film peut être réalisé complètement au sein d'un studio, initié par un producteur ou réalisé par un studio externe travaillant « à façon », ou initié par un producteur qui monte une équipe ad hoc pour le réaliser. Du point de vue des organisations, on observe trois grandes configurations dans la prise en charge de l'activité de création, qui se traduisent aussi par des enjeux managériaux différents.

\section{La direction créative}

Cette première configuration repose sur le modèle d'une organisation développée comme un outil - appendice, appareil pour porter les projets d'un créateur interne à l'organisation. Il s'agit d'un modèle

4. C'est la frontière qui distingue, dans le champ de l'innovation, la conception réglée qui renvoie à des logiques d'innovation incrémentale de la conception innovante qui renvoie à des logiques d'innovation disruptive. 
intégré qui permet de matérialiser la vision du créateur dans un ensemble de processus, de routines ou d'artefacts permettant de coordonner le travail des équipes de création. On retrouve ce modèle dans les secteurs comme la gastronomie et l'architecture où le créateur en tant qu'individu incarne le plus souvent l'entreprise (qui porte parfois son nom) tout en collaborant avec des équipes qui travaillent au déploiement de sa vision. Dans une étude d'un restaurant gastronomique, Bouty et Gomez (2010) montrent qu'une grande partie du travail de génération d'idées et développement de nouveaux plats est prise en charge par le chef, une autre part est effectuée par les seconds et très peu par les cuisiniers. Cette configuration pose la question de la transmission de la vision intime et subjective du créateur et demande à l'organisation de gérer un équilibre entre créativité et standardisation à travers une forme de « codification» assurant la reproduction de la création par d'autres que le créateur (Slavich et al., 2014). Suivant les secteurs et les entreprises, l'ensemble du processus de création peut être pris en charge presque totalement par les équipes, seuls les choix créatifs restent toujours la primauté du créateur. La direction créative est une configuration qui pose néanmoins de forts enjeux managériaux liés à la dépendance au créateur, lors de sa succession par exemple (Paris et Leroy, 2014).

\section{L'orchestration de contributions créatives hétérogènes}

Dans cette deuxième configuration, une organisation est constituée autour de différentes ressources qu'elle mobilisera avec d'autres contributions externes, multiples et hétérogènes, dans le cadre d'un projet de création. Les créateurs sont le plus souvent externes à l'entreprise et l'organisation assure la constitution des équipes créatives. On retrouve ce modèle dans l'édition de livres, de mobilier ou d'objets de design, dans la production de musique ou de spectacles. Dans une étude des producteurs de musique country de Nashville, Lingo et O'Mahony (2010) soulignent l'importance $\mathrm{du}$ rôle de broker (ou courtier) des producteurs, dont l'activité consiste à réunir (ou garder à l'écart) certains collaborateurs autour d'un projet. Le Cirque du Soleil illustre cette configuration où l'entreprise invite un metteur en scène externe à collaborer à un projet de spectacle tout en le mettant en relation avec un ensemble de concepteurs, d'artistes et des ressources pouvant être internes ou externes à l'entreprise (Massé et Mirc, 2020). L'organisation œuvre donc principalement à l'orchestration de ces différentes ressources au cours du processus de création.

\section{Facilitation de l'émergence de projets créatifs}

La troisième configuration renvoie à des organisations qui font émerger des projets portés par divers créateurs, et les produisent en interne. Cette configuration se retrouve principalement dans de grandes organisations, structurées en projets autour de créateurs internes. L'organisation joue ici un rôle important dans la mise en place d'un climat favorable à la créativité, en promouvant l'émergence de nouveaux projets créatifs et en manageant les étapes du processus créatif. Ces enjeux renvoient plus directement à la littérature sur la créativité organisationnelle (Amabile, 1988; Woodman et al., 
1993) et semblent plus directement transposables dans les divers contextes industriels et organisationnels. Pixar constitue l'un des exemples emblématiques de cette configuration, dans laquelle l'entreprise s'est constituée en terreau qui permet à divers réalisateurs internes à l'entreprise de proposer des projets audacieux et de les développer (Catmull et Wallace, 2014).

Si certaines configurations sont prédominantes dans certains secteurs ou types d'entreprises, elles peuvent cohabiter dans un même secteur. Certaines organisations donnent par ailleurs lieu à des configurations plus complexes, la direction des projets n'étant rarement l'apanage d'une seule personne. À titre d'exemple, dans le domaine des jeux vidéo, l'entreprise Ubisoft s'inscrit dans la configuration de facilitation, l'entreprise favorisant l'émergence de nouveaux projets de jeux par une grande liberté créative donnée à ses 45 studios répartis dans le monde (Chiambaretto et al., 2019). Néanmoins, l'enjeu de maîtrise du risque sur les projets donne lieu à un ensemble de dispositifs de contrôle, qui impliquent aussi des outils et principes en matière de création (Lê et al., 2013 ; Massé, 2019). La répartition entre des studios en charge de la création et une structure administrative chargée du suivi se révèle ainsi toute théorique, dans la mesure où la mise en place d'un cadre pour la création peut difficilement se faire sans connotation éditoriale. Dans le cas présent, l'entreprise assumait d'ailleurs le fait d'avoir des projets portés par les studios, mais dans le cadre d'une ligne éditoriale portée par l'entreprise.

Ainsi, les configurations évoquées constituent-elles des archétypes, qui ne se retrouvent pas toujours aussi nettement dans la nature. Cela nous ramène à la dimension collective de la création et parfois diffuse de la décision éditoriale. Et cela souligne par ailleurs la question fondamentale de l'articulation entre la création et le contrôle. La finesse du réglage entre ces dimensions se révèle critique. Elle montre que la création doit être vue comme un processus qui fait la part belle à la confrontation, à la négociation, au compromis et à l'ajustement. Les tensions ou paradoxes mis en avant par la littérature sont l'essence même de l'acte de création, comme rencontre entre une intention idéelle et un substrat réel.

\section{IV - PRÉSENTATION DES CONTRIBUTIONS DES AUTEURS À CE DOSSIER}

Les articles de ce dossier portent sur des secteurs et contextes aussi différents que la musique, le design, la mode, l'opéra et le cinéma. Certains évoluent dans la sphère marchande, d'autres dans la sphère subventionnée, certains ont une dimension de reproduction industrielle, d'autres non, certains entrent dans une économie du service quand d'autres reposent sur la vente de produits. Néanmoins, tous partagent des problématiques communes liées à l'activité de création. Les angles proposés et problématiques de gestion varient aussi : il peut s'agir de construction de réseau, de gestion de production, de constitution d'équipes. Tous mettent en avant un lien important entre ces considérations de gestion et les projets artistiques sur lesquels elles portent. L'ordre de présentation des numéros suit une logique de diminution de focale, du plus macro au plus micro.

Dans le premier article, Céline Bourbousson, Jean-Marie Furt et Anne Iglésias s'intéressent à l'émergence d'une dynamique 
d'entrepreneuriat collectif à travers l'étude de créateurs corses participants au festival Creazione (design et mode). Sur la base d'une analyse multi-niveaux, les résultats font émerger une dynamique de confrontation et d'articulation des logiques commerciale, éthique territoriale et artisanale au niveau méso. Cette étude permet d'enrichir la compréhension des tensions identitaires vécues par les créateurs.

Albéric Tellier explore l'ouverture des processus de création en étudiant des collectifs d'acteurs dans le rap américain. L'article analyse la production de 22 albums de rap classés dans les 10 premières places du classement « Billboard 200 ». À travers l'analyse de réseaux de type «cœur/périphérie », l'auteur souligne que ces collectifs mobilisent des membres de différents types de communautés permettant d'activer des processus d'intégration (« outside-in ») et de valorisation externe (« inside-out»). L'article contribue à la compréhension de la configuration de l'orchestration et montre que l'ouverture du processus de création a des conséquences sur les caractéristiques des œuvres produites notamment via une forme d'homogénéisation de l'offre, et que les acteurs semblent devoir évoluer dans des réseaux de relations pour participer au processus créatif.

Ensuite, Nathalie Schieb-Bienfait, Sandrine Emin et Danielle Pailler analysent l'émergence de collectifs créatifs engagés dans la restauration du lien entre la dimension créative et la dimension sociale de leur activité. L'article explore deux études de cas de l'univers de la musique (les collectifs initiés par Sébastien Boisseau et le collectif «Des Liens » initié par Dominique A.) et décrit des processus d'émergence d'ouverture à d'autres parties prenantes en trois temps : l'initiation, l'activation du collectif, et la routinisation. Ces collectifs qui peuvent mobiliser un large éventail d'acteurs (artistes, mais aussi des acteurs d'autres univers de travail comme le social, la santé, l'éducation, etc.) cherchent à reconfigurer des modes de production, de circulation et d'échange entre les univers créatifs et la société.

Dans le quatrième article, Agnès MazarsChapelon et Fabienne Villesèque-Dubus s'intéressent aux spécificités du contrôle et du pilotage dans les industries créatives à travers l'étude d'un cas du secteur du film numérique. Cet article contribue à affiner l'analyse des résistances des entreprises au paradigme de la création en montrant que les outils de contrôle et de pilotage peuvent aussi s'adapter à l'activité de création. Afin de gérer la forte incertitude et les contraintes de temps du projet créatif, les auteurs mettent en lumière deux dimensions que recouvrent les activités de pilotage et de contrôle : une combinaison d'outils financiers et non financiers spécifiques pour gérer la contrainte temporelle du projet et la mise en place d'outils hybrides permettant une gestion simultanée de la tension temporelle et de l'incertitude artistique inhérente au caractère prototypique du produit et plus largement à l'activité de création. Cette configuration permet de piloter le résultat de projet de création, mais également les ressources et les compétences à mobiliser dans le processus de création.

En clôture de ce dossier, Camille de Bovis, Caroline Hussler et Anne-Sophie Barbe examinent le rôle des artefacts et de leurs concepteurs dans le processus de création d'un opéra. Cette perspective micro du processus de création a pour objectif de mieux comprendre les mécanismes de 
coordination entre créateurs/concepteurs et performeurs, phénomène central dans la configuration de direction créative présentée précédemment. À travers une étude ethnographique qui interroge le processus de création au niveau micro, les auteurs décrivent le rôle de la conduite d'opéra (artefact qui encapsule l'esprit d'une œuvre originale) et les activités du régisseur de scène. L'étude met en lumière trois mécanismes par lesquels les concepteurs d'artéfacts peuvent aligner les pratiques effectives des utilisateurs d'un artefact avec l'abstraction qu'ils ont tenté d'y encoder.

Pour terminer, nous adressons tous nos remerciements à l'ensemble des auteurs pour leurs contributions, ainsi qu'à l'ensemble des collègues qui ont, en proposant un projet d'article, contribué à enrichir ce champ de recherche. Ce dossier spécial a pu être réalisé grâce au précieux travail des évaluateurs qui ont ainsi contribué à l'amélioration des articles retenus. Nous tenons ainsi à remercier Alban Ouahab, Albane Grandazzi, Anne Gombault,
Barbara Slavich, Bérangère Szostak, Charlène Arnaud, Christophe Midler, Colette Depeyre, Elen Riot, Emmanuel Coblence, Eve Lamendour, Florence Charue-Duboc, Frédéric Kletz, Frédéric Leroy, Gilles Garel, Héloïse Berkowitz, Jérémy Morales, Julia Parigot, Lise Gastaldi, Louis-Etienne Dubois, Margot Leclair, Marie-Léandre Gomez, Marine Agogué, Mathias Béjean, Nicolas Aubouin, Olivier Germain, Philippe Bouquillion, Raphaël Suire, Rémi Maniak, Romain Gandia, Romaric Servajean-Hilst, Sandrine Emin, Serge Poisson De Haro, Sylvain Bureau, Thierry BurgerHelmchen.

Nous tenons également à exprimer notre gratitude au comité éditorial de la Revue française de gestion, en particulier à JeanPhilippe Denis, rédacteur en chef, à Nathalie Dubost, rédactrice en chef adjointe et à Alice Rouquié, secrétaire de rédaction aux Éditions Lavoisier. Leur confiance et leur accompagnement durant le processus éditorial ont permis de préparer ce dossier dans de très bonnes conditions.

\section{BIBLIOGRAPHIE}

Abecassis-Moedas C. et Benghozi P.J. (2012). "Efficiency and innovativeness as determinants of design architecture choices", Journal of Product Innovation Management, vol. 29, n⿳ 3 , p. 405-418.

Agid P. et Tarondeau J.-C. (2003). « L’Opéra de Paris est-il économiquement gouvernable ? », Revue française de gestion, vol. 29, n 142, p. 147-168.

Amabile T. (1988). Creativity in Context, Westview Press, Boulder, Colo.

Becker H.S. (1974). “Art as collective action”, American Sociological Review, vol. 39, $\mathrm{n}^{\mathrm{o}} 6$, p. 7767-7776

Becker H.S. (1982). Art Worlds, University of California Press, Berkeley.

Benghozi P.-J. (1995). «Les sentiers de la gloire : savoir gérer pour savoir créer », Des Savoirs En Action, Contribution de la recherche en gestion, logiques gestion, L'Harmattan, Paris. 
Benghozi P.-J. et Paris T. (2003). « De l'intermédiation à la prescription : le cas de la télévision », Revue française de gestion, vol. 29, n ${ }^{\circ}$ 142, p. 205-227.

Bilton C. (2006). Management and Creativity: From Creative Industries to Creative Management, Wiley-Blackwell, Malden.

Bouty I. et Gomez M.-L. (2010). « Des ressources à la pratique », Revue française de gestion, vol. $36, n^{\circ} 203$, p. 149-161.

Camdessus M. (2004). Le sursaut : vers une nouvelle croissance pour la France, Rapport officiel, Documentation française, Paris.

Catmull E. et Wallace A. (2014). Creativity, Inc: Overcoming the Unseen Forces that Stand in the Way of True Inspiration, Random House.

Cattani G., Ferriani S., Negro G. et Perretti F. (2008). "The structure of consensus: Network ties, legitimation, and exit rates of U.S. feature film producer organizations", Administrative Science Quarterly, vol. 53, n ${ }^{\circ}$ 1, p. 145.

Caves R. (2000). Creative Industries: Contracts Between Art and Commerce, Harvard University Press, Cambridge, Mass.

Chiambaretto P., Massé D. et Mirc N. (2019). "All for one and one for all? Knowledge broker roles in managing tensions of internal coopetition: The Ubisoft case", Research Policy, vol. $48, \mathrm{n}^{\mathrm{o}} 3$, p. $584-600$

Cohen D. et Verdier T. (2008). La mondialisation immatérielle, rapport, Les rapports du Conseil d'analyse économique, Documentation française, Paris.

Cohendet P., Grandadam D. et Simon L. (2010). "The anatomy of the creative city", Industry \& Innovation, vol. $17, \mathrm{n}^{\mathrm{o}} 1, \mathrm{p} .91-111$.

Cohendet P. et Simon L. (2007). "Playing across the playground: Paradoxes of knowledge creation in the videogame firm", Journal of Organizational Behavior, vol. 28, $\mathrm{n}^{\circ}$ 5, 587, p. $91-111$.

DCMS (2001). Creative Industries Mapping Document. UK Government Department for Culture, Media and Sport.

DCMS (1998). Creative Industries Mapping Document. UK Government Department for Culture, Media and Sport.

Department of Communications and the Arts (1994). Creative Nation: Commonwealth Cultural Policy, Department of Communications and the Arts, Canberra, Australia.

Durand R., Rao H. et Monin P. (2007). "Code and conduct in French cuisine: Impact of code changes on external evaluations", Strategic Management Journal, vol. 28, nº 5, p. 455-472.

Endrissat N., Islam G. et Noppeney C. (2016). "Visual organizing: Balancing coordination and creative freedom via mood boards", Journal of Business Research, vol. 69, n ${ }^{\circ} 7$, p. 455-472.

France Créative (2013). Panorama des industries culturelles et créatives - Au coeur du rayonnement et de la compétitivité de la France, France Créative, Paris. 
Glynn M.A. (2000). "When cymbals become symbols: Conflict over organizational identity within a symphony orchestra”, Organization Science, vol. 11, no 3, p. 285-298.

Jones C. et Livne Tarandach R. (2008). "Designing a frame: Rhetorical strategies of architects", Journal of Organizational Behavior, vol. 29, nº 8, p. 1075-1099.

KEA (2009) The Impact of Culture on Creativity, Study for European Commission, Directorate-General for Education and Culture.

Lampel J.Lant T. et Shamsie J. (2000). "Balancing act: Learning from organizing practices in cultural industries", Organization Science, vol. 11, n 3, p. 2633.

Lê P., Massé D. et Paris T. (2013). "Technological change at the heart of the creative process: Insights from the videogame industry". International Journal of Arts Management, vol. 15, $\mathrm{n}^{\mathrm{o}} 2$, p. 45-60.

Lévy M. et Jouyet J.-P. (2006). L'économie de l'immatériel : la croissance de demain, Rapport de la commission sur l'économie de l'immatériel, ministère de l'Économie, des Finances et de l'Industrie, Paris.

Lingo E.L. et O'Mahony S. (2010) "Nexus work: Brokerage on creative projects", Administrative Science Quarterly, vol. 55, $\mathrm{n}^{\mathrm{o}}$ 1, p. 47-81.

Mainemelis C., Kark R. et Epitropaki O. (2015). "Creative leadership: A multi-context conceptualization", Academy of Management Annals, vol. 9, nº 1, p. 393-482.

Massé D. (2019). "The routines of creation: From artistic direction to collective exploration", Management of Extreme Situations: From Polar Expeditions to Exploration-Oriented Organizations, Lièvre P., Aubry M., Garel G. (Eds), John Wiley \& Sons, Hoboken, NJ, p. 323-338.

Massé D. et Mirc N. (2020). "Knowledge brokers to contain ambidextrous tensions", AIMS 2020, en ligne, 2-5 juin.

Massé D. et Paris T. (2013). «Former pour entretenir et développer la créativité de l'entreprise : les leçons du Cirque du Soleil », Gestion, vol. 38, n 3, p. 6.

Menger P.-M. (2009). Le travail créateur : S'accomplir dans l'incertain, Edition du Seuil, Paris.

Midler C. (1986). « La logique de la mode managériale », Gérer et Comprendre-Annales des Mines, $\mathrm{n}^{\mathrm{o}} 3$, p. 74-85.

Moeran B. (2009). "The organization of creativity in Japanese advertising production", Human Relations, vol. 62, $\mathrm{n}^{\mathrm{o}} 2$, p. 963-985

Paris T. (2013). «Des mariages féconds ? Comprendre la diversité des modes d'organisation de la création », Les annales des Mines, Gérer et Comprendre, n ${ }^{\circ} 113$, p. 30-39.

Paris T. (2010). Manager la créativité : Innover en s'inspirant de Pixar, Ducasse, les Ateliers Jean Nouvel, Hermès..., Pearson Village Mondial, Paris.

Paris T., Lang G. et Massé D. (2020). "Polarized worlds and contextual creativity in creative industries: The case of creation processes in the perfume industry", International Management, vol. 24, $\mathrm{n}^{\mathrm{o}}$ 2, p. 12-26. 
Paris T. et Leroy F. (2014). "Managing transition in an artistic company with entrepreneurial Management: A case study of Groupe Bernard Loiseau", International Journal of Arts Management, vol. 16, p. 42-54.

Slavich B., Cappetta R. et Salvemini S. (2014). "Creativity and the reproduction of cultural products: The experience of Italian haute cuisine chefs", International Journal of Arts Management, vol. 16, $\mathrm{n}^{\mathrm{o}}$ 2, p. 29-41.

Svejenova S., Planellas M. et Vives L. (2010). “An individual business model in the making: A chef's quest for creative freedom”, Long Range Planning, vol. 43, n 2-3, p. 408-430.

Tschang F.T. (2007). "Balancing the tensions between rationalization and creativity in the video games industry”, Organization Science, vol. 18, nº 6, p. 989.

Thompson P., Jones M. et Warhurst C. (2007). "From conception to consumption: Creativity and the missing managerial link", Journal of Organizational Behavior: The International Journal of Industrial, Occupational and Organizational Psychology and Behavior, vol. 28, $\mathrm{n}^{\mathrm{o}} 5$, p. 625-640.

UNCTAD (2010). Creative Economy, United Nations Conference on Trade and Development, Genève.

Verganti R. (2003). "Design as brokering of languages: Innovation strategies in Italian firms", Design Management Journal, vol. 14, n 3, p. 34-42.

Woodman R.W., Sawyer J.E. et Griffin R.W. (1993). "Toward a theory of organizational creativity", The Academy of Management Review, vol. 18, n 2, p. 93-321. 